\title{
Setting up a transoesophageal echocardiography service
}

\author{
S Saltissi, M A de Belder, P Nihoyannopoulos
}

Department of Cardiology, Royal Liverpool University Hospital, Liverpool

S Saltissi

\section{Regional}

Cardiothoracic

Centre, South

Cleveland Hospital,

Middlesbrough,

Cleveland

$M$ A de Belder

Department of

Cardiology,

Hammersmith

Hospital, London

P Nihoyannopoulos

Correspondence to:

Dr S Saltissi, Department of Cardiology, Royal Liverpool University Hospital, Presco Street, Liverpool L7 8XP
Transoesophageal echocardiography (TOE) provides better information on cardiac anatomy and function than conventional transthoracic echocardiography (TTE) because of the proximity of posterior cardiac structures to the oesophagus containing the TOE probe and the absence of intervening bone and/or lung (figs 1 and 2). The clarity of the resulting images, Doppler spectra, and colour flow maps (figs 3 and 4) improves their subjective interpretation in a wide range of conditions and increases confidence in the diagnosis.

TOE undoubtedly requires considerable extra investment of time and money in equipment, staff, and training. Each hospital must weigh this against the clinical value of the information obtained, the likely number of patients who may benefit from the test, and the availability of a similar local service. This balance depends on the clinical relevance of the technique and the case mix of patients: it will be different in district general hospitals (DGH), cardiothoracic units, and research based echocardiography departments. It is generally accepted that cardiothoracic units and research departments should have on-site TOE, but not as yet that all DGHs need to have TOE, particularly when this technology is available nearby. None the less, I believe that all large, busy DGHs that have one or more cardiologists (or physicians with an interest in cardiology) should have on-site TOE.

\section{Indications}

There are two main reasons for a TOE examination (table 1). Firstly, it is impossible to obtain an adequate TTE examination in 10\% to $20 \%$ of patients, and in these patients TOE is the only means of obtaining echocardiographic data. Secondly, even when the TTE images are technically good, TOE provides additional information because the quality of the image is improved, because structures visible on the TTE (particularly posteriorly located ones such as the left atrium and mitral valve) are shown in more detail, or because TOE provides images of structures not visualised by TTE, such as the left atrial appendage and pulmonary veins. Group A indications (table 1) are common and relevant to all institutions including DGHs and group B indications are likely to be more common in cardiothoracic units than in DGHs. Group C indications are currently important only in echocardiographic research units.

\section{Equipment requirements}

Table 2 lists the equipment required for a TOE service.

A unit planning a TOE service will already have an echocardiograph with $M$ mode, cross
Figure 1 Relation between the heart, great vessels, and oesophagus. The transoesophageal transducer is shown at two different levels within the oesophagus together with the resulting image planes obtained. Ao, aorta; $L A$, left atrium; $L A A$, left atrial appendage; LUPV, left upper pulmonary vein. $L V$, left ventricle; $M V$, mitral valve; $P V$, pulmonary valve; $R A$ right atrium; $R U P V$, right upper pulmonary vein; $R V$, right ventricle; SVC, superior vena cava; $T V$, tricuspid valve.

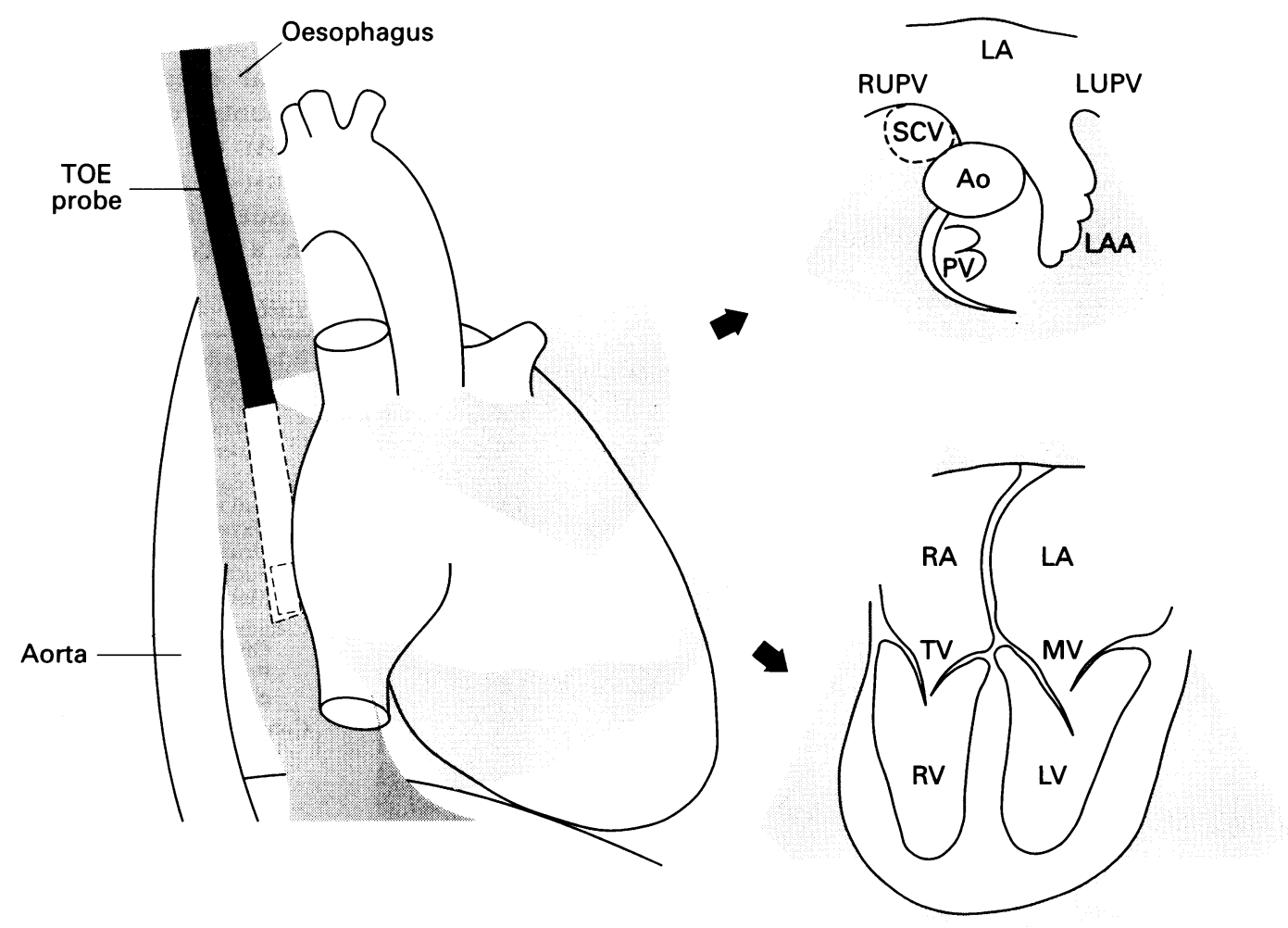




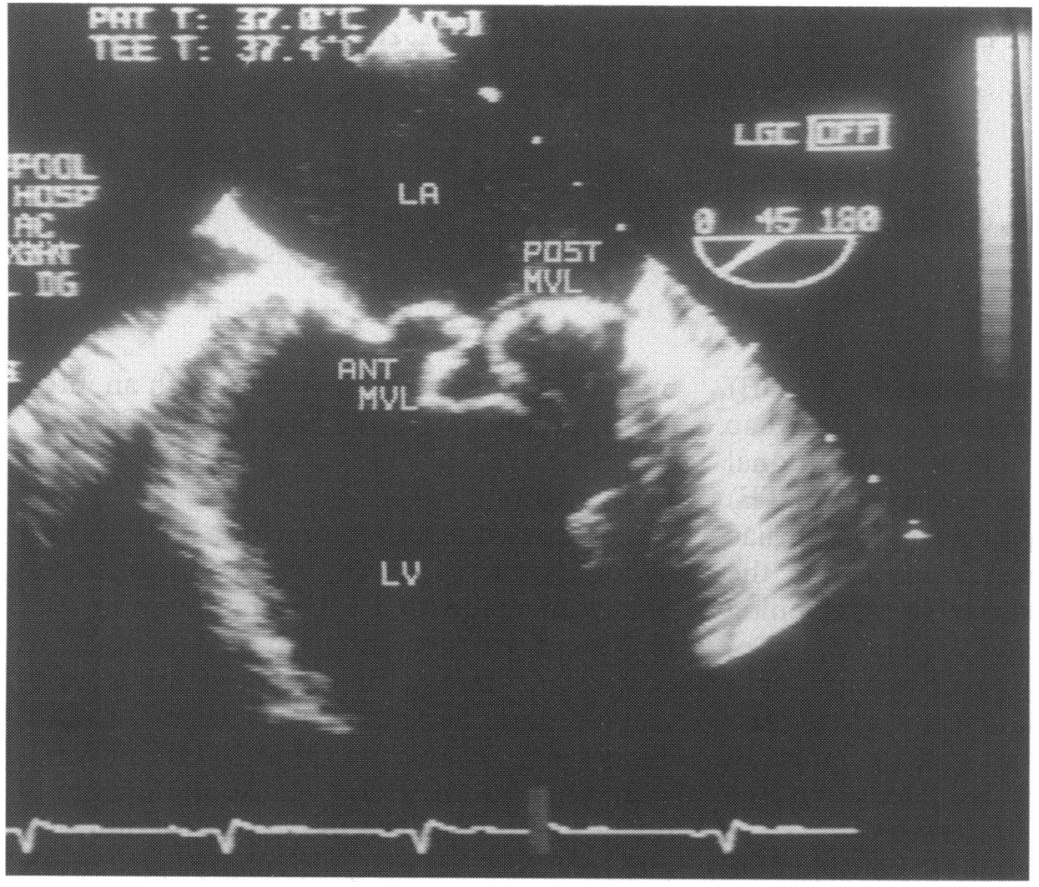

Figure 2 Short axis view of mitral valve obtained with a multiplane transducer. The anatomy of the prolapsing, floppy anterior and posterior mitral valve leaflets is very clearly seen. ANT, anterior; POST, posterior; MVL, mitral valve leaflet. See legend to fig 1 for other abbreviations.

sectional, and Doppler facilities. Most manufacturers of modern echocardiographic equipment produce or recommend one or more compatible TOE probes. Until recently only single (transverse) plane imaging probes were available but now biplane probes-that is those imaging in the longitudinal and transverse axes-and multiplane probes are increasingly becoming available. Such probes allow structure to be seen from different orientations and so provide a better spatial appreciation in three dimensions of any abnormality and of the relation of one structure to another. This is useful in conditions such as congenital heart disease and aortic

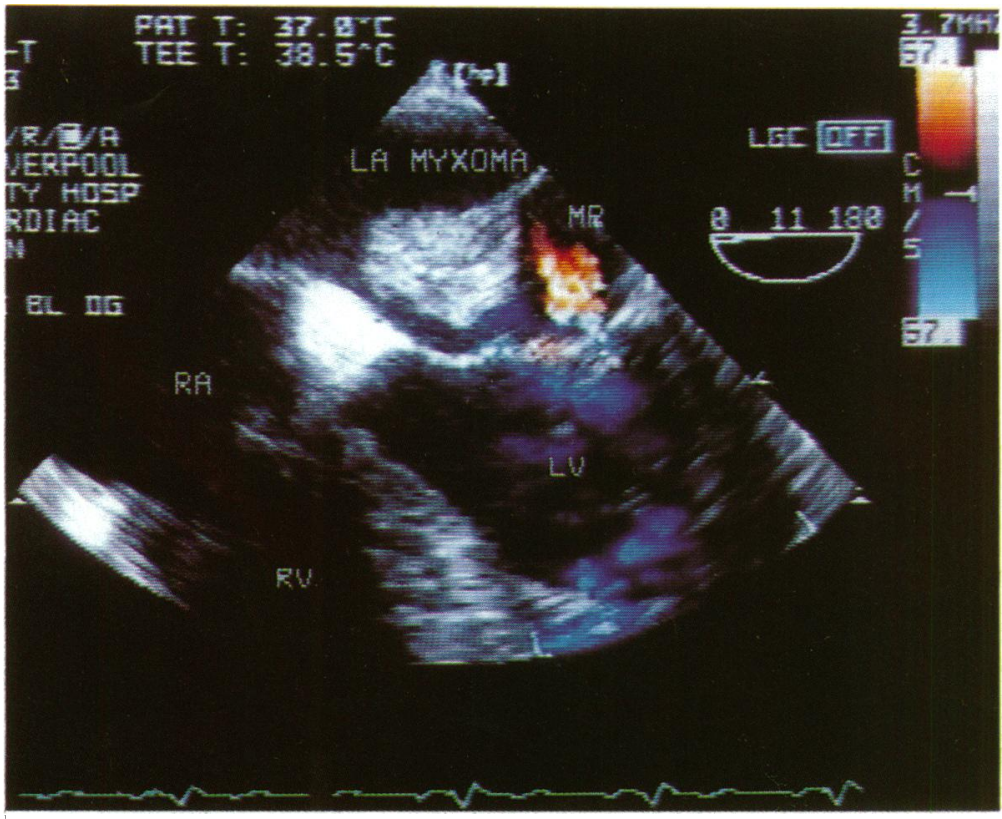

Figure 3 Transoesophageal four chamber view showing a large left atrial myxoma. A jet of mitral regurgitation is clearly seen on the colour flow map. MR, mitral regurgitation. See legend to fig 1 for other abbreviations.
Table 1 Indications for TOE

\section{Inadequate/impossible TTE}

Adverse patient characteristics - for example, gross obesity or marked pectus excavatum. Coexisting disorder-for example, chronic obstructive pulmonary disease, chest trauma. Inconvenient patient location-for example, ITU, ventilation, haemodialysis unit.

Additional information required

Group A

$$
\text { Additional information required }
$$

Infective endocarditis and its complications

Cardiac site for embolism

Clarification of intracardiac masses

Native valve structure-for example, tears and prolapse Thoracic aortic disease-for example, dissection, aneurysm, atherom

Group B:

Prosthetic valve structure and function-

for example, leaks, thrombus

Adult congenital heart disease-for example, ASD

During cardiac surgery-for example, MV repair, HOCM

During non-cardiac surgery, mainly for LV function

On ITU, mainly for LV function

Group C:

Diagnosis of IHD-pacing techniques

3D image reconstruction

Tissue characteristics

ITU, intensive therapy unit; $M V$, mitral valve; ASD, atria septal defect; HOCM, hypertrophic obstructive cardiomyopathy; LV, left ventricular; IHD, ischaemic heart disease.

dissection and for the assessment of prosthetic valves.

Because the patient lies in the left lateral position for up to 45 minutes a comfortable couch or trolley is needed. Ideally this should have lockable wheels, be of adjustable height, and have an adjustable head section. More than one couch/trolley will be needed if several studies are performed in the same session.

TOE is a safe procedure but serious complications can develop (see later). This means that equipment to monitor vital signs, including blood pressure and the electrocardiogram ECG and very occasionally pulse oximetry for oxygen saturation, must be available. Full resuscitation equipment including a "crash" trolley and defibrillator must also be immediately available. Saliva and upper respiratory tract secretions need to be aspirated through a suction apparatus. Patients occasionally need oxygen and this can be supplied through a nasal speculum from an oxygen cylinder mounted on the trolley.

At the end of the procedure, the probe must be removed, rinsed, and cold sterilised (usually in $2 \%$ glutaraldehyde solution) either in a dedicated covered tank or in a cold

\section{Table 2 Equipment needed for TOE}

Echo hardware:

Cross sectional machine with video and hardcopy facility TOE probe

Instrument trolley

Patient couch/trolley

Safety equipment:

Full resuscitation trolley with defibrillator

Oxygen source

Drugs and infusion fluids

Suction apparatus

Monitors-for example, BP cuff, ECG machine, oximeter

Cleaning equipment and materials:

Glutaraldehyde tank/cold steriliser Protective clothing
gloves and goggles

Consumables:

Gauze swabs

Lubricant gel

Gloves, aprons, patient gowns

ECG leads

Bite guards

Cleansing solutions

Protective rubber sheaths for probes (optional)

BP, blood pressure. 


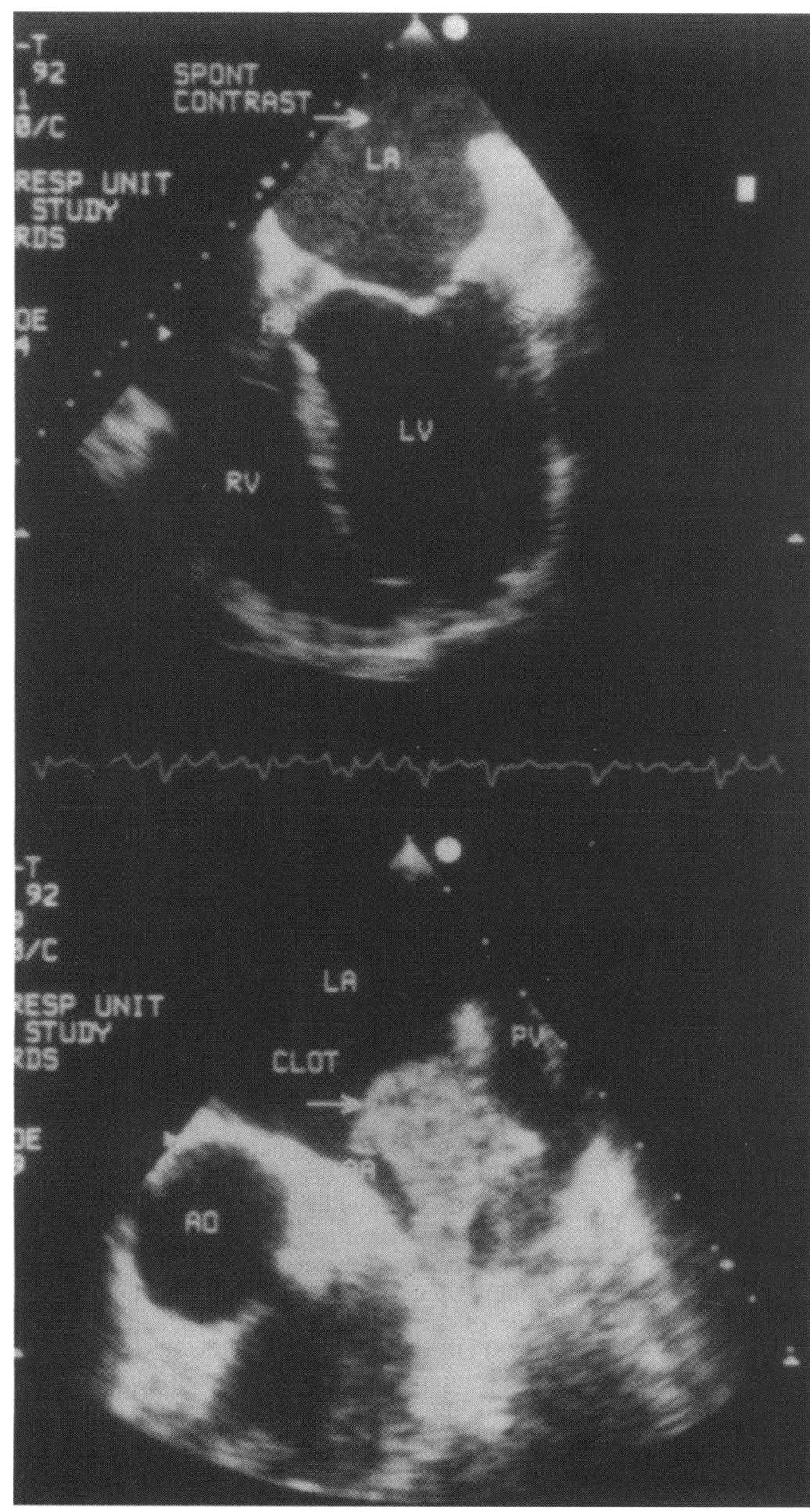

Figure 4 Transoesophageal images obtained with a single (transverse) plane transducer. The transthoracic images in this patient presenting with a transient ischaemic attack were of good quality and entirely normal. Top: Four chamber view showing pronounced spontaneous echo contrast in the left atrium. Bottom: Transverse axis image at the level of the left atrium. A large pedunculated thrombus is clearly shown in the left atrial appendage. PV, pulmonary vein; $A O$, aorta. See legend to fig 1 for other abbreviations.

steriliser according to manufacturer's directions. The staff should be protected against skin and eye sensitisation to glutaraldehyde by rubber gloves, goggles, and gowns. At the end of the session the probe must be stored according to manufacturer's recommendations either by hanging from a rack or in an individual storage case.

\section{Location}

TOE equipment requires a large laboratory. The gastroenterology (GI) endoscopy suite or the echocardiography laboratory are suitable sites for TOE. The GI endoscopy suite has cleaning and storage facilities, medications, monitoring equipment, nurses trained in endoscopy, and endoscopists to help with diffcult intubations. Echocardiographic equipment, however, will have to be moved and a full GI programme will lead to an inflexible schedule. The echocardiography laboratory is convenient, has readily available echocardiographic equipment, and enables easy scheduling, though additional equipment for monitoring, cleaning, storage, and suction may need to be bought. Either location is appropriate but in practice most units prefer the familiarity of the echocardiography laboratory.

Whichever site is chosen, an adjacent area is needed for cleaning, disinfecting, and storing the transducer probe. To fulfil Health and Safety Executive regulations the dimensions and ventilation of this room need to keep the atmospheric concentration of glutaraldehyde below set limits during cleaning. These requirements must be discussed with the local designated health and safety representative who may monitor concentrations.

Occasional urgent TOE investigations can be performed almost anywhere including accident and emergency departments, intensive therapy units, coronary care units, or the cardiac catheter laboratory. These areas, however, are not suitable as a permanent site for TOE facilities.

\section{Staffing}

A physician, cardiac technician, and a nurse (or further technician) are required for TOE (table 3). The TOE operator will usually be a cardiologist or physician with cardiac training who is experienced in probe introduction and manipulation and familiar with the controls on the probe and echocardiograph. Before starting, however, the operator must ensure that the TOE investigation is both indicated and appropriate and that there are no contraindications. Then he or she must fully explain the procedure to the patient (even if an assistant has already done so). This is to allay any fears and to reassure the patient that the test is noninvasive. No drug is as anxiolytic as a calm, confident, informative, and competent operator. Indeed, some units prefer not to sedate patients because they believe that explanation and instruction are sufficient. Most units in the United Kingdom, however, (including mine) almost always give a small dose of sedative (usually midazolam) as much for its amnesic properties as for its sedative effects. The operator must obtain written consent for the investigation and any possible additional procedures (before sedation if used). A functioning intravenous line must be in place: I advise using a medium sized cannula in a proximal vein-for example, the antecubital fossa-so that contrast can be injected if necessary.

Safe TOE examination requires two more health care workers in addition to the operator. A cardiac ultrasound technician is needed to set up the echocardiogram and probe and to alter the gain controls, image depth, and 
Patient checklist for TOE

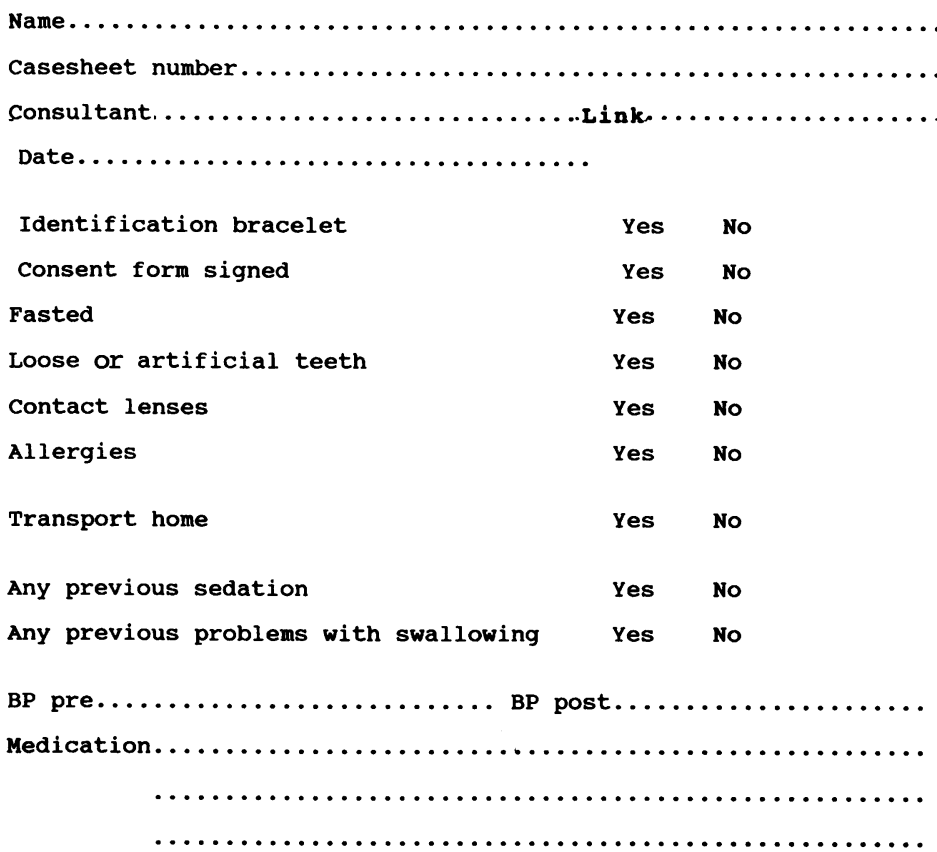

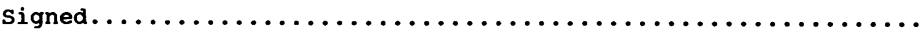

Figure 5 Checklist on patient data for transoesophageal echocardiography.

\begin{tabular}{l} 
Table 3 Staff roles in TOE \\
\hline Physician operator \\
Before: \\
Explanation of the procedure \\
Obtaining patient consent \\
Establishing IV access and administering drugs \\
During: \\
Performing the examination \\
After: \\
Interpretation and reporting of results \\
Technician \\
Before: \\
Setting up echo machine and probe \\
During: \\
Driving echo machine \\
Image recording/hard copy \\
Image measurement/interpretation \\
Nurse (or technician) \\
Before: \\
Room set up/equipment check \\
Patient check list \\
Patient positioning \\
During: \\
Aiding intubation \\
Monitoring vital signs \\
Oral suction of secretions \\
Administration of oxygen and/or drugs \\
After: \\
Overseeing recovery \\
Cleansing and disinfecting \\
Discussion with patients and relatives \\
\hline IV, intravenous.
\end{tabular}

Table 4 Operator skills for TOE

\begin{tabular}{llll}
\hline Component & Objective & Duration & No of cases \\
\hline $\begin{array}{l}\text { General echocardiography } \\
\text { (USA Level 11) }\end{array}$ & $\begin{array}{l}\text { Background for } \\
\text { performance } \\
\text { and interpretation }\end{array}$ & $\begin{array}{l}\text { Minimum } \\
\text { T mnth }\end{array}$ & 300 \\
Oesophageal intubation & $\begin{array}{l}\text { TOE probe } \\
\text { insertion }\end{array}$ & Variable & 25 \\
TOE examination technique & $\begin{array}{l}\text { TOE performance } \\
\text { and interpretation } \\
\text { Maintenance of competence }\end{array}$ & Variable & Annual \\
Ongoing education & 50 & $50-75$ \\
\hline
\end{tabular}

colour flow mapping during the procedure and so exert an element of quality control. Some units record the entire procedure on video for later editing and analysis whereas in others the technician is required both to edit in real-time (by recording only relevant parts of the procedure) and also to generate appropriate hard copy if necessary. Technicians quickly become conversant with image interpretation and are invaluable, frequently bringing to the attention of the operator some features of the image that otherwise might have been missed.

A third team member (table 3), either an endoscopy nurse or an additional cardiac technician appropriately trained in endoscopy techniques, is needed to complete the patient checklist (fig 5), make the patient comfortable, explain the procedure, and reassure the patient. Patients tend to fear the unknown. They are scared of being unable to swallow the probe and of pain during the procedure. Time spent on explanation and reassurance can allay these fears and prevent a petrified, uncooperative patient from fulfilling his own prophecy.

During the procedure the operator relies on these assistants to monitor vital signs, intermittently aspirate copious oral secretions, which can impair breathing, and alert him or her to any emerging complications or problems. Occasionally the assistant will be required to administer oxygen or even drugs as instructed.

After the procedure the patient is usually wheeled to a recovery area and observed by one of the assistants. Patients must not be allowed to leave until they are fully conscious, able to swallow, and free of complications. After sedation patients often hear little and remember less of what is said to them so it is good practice for the assistant to talk to any relatives present (as well as to the patient) to give further safety instructions and some indication of the findings of the test (after discussion with the operator) and to ensure that follow up arrangements are adequate. Finally, one of the assistants will clean and disinfect the TOE probe and set up the laboratory and the equipment for the next investigation.

\section{Physician training}

To perform the tasks outlined in the preceding section the operator needs considerable knowledge and skills. Tables 4 and 5 summarise guidelines published by the American Society of Echocardiography for the training of US physicians in TOE. ${ }^{1}$ It seems reasonable to expect similar standards of investigators in the United Kingdom.

Operators should start from a baseline of being competent and experienced in general TTE (table 4). Then they need to become proficient in oesophageal intubation and to learn the additional skills essential to the TOE examination. Competence must be maintained by continuing education and by doing at least 50-75 studies a year.

The specific additional skills necessary for TOE (table 5) include knowledge of cardiac anatomy as it relates to various cross sectional planes in health and disease; familiarity with artefacts; and an understanding of the indica- 
Table 5 Acquisition of operator skills for TOE

\begin{tabular}{ll}
\hline Skill & Source \\
\hline $\begin{array}{l}\text { Cognitive: } \\
\text { Indications/contraindications }\end{array}$ & $\begin{array}{l}\text { Textbooks } \\
\text { Risks }\end{array}$ \\
$\begin{array}{l}\text { Cross sectional anatomy } \\
\text { Interpretations of images }\end{array}$ & $\begin{array}{l}\text { Videos } \\
\text { Observations } \\
\text { PM room }\end{array}$ \\
$\begin{array}{l}\text { Practical: } \\
\quad \text { Probe introduction }\end{array}$ & GI lab \\
$\begin{array}{l}\text { Probe manipulation } \\
\text { Optimisation of controls }\end{array}$ & $\begin{array}{l}\text { Observation } \\
\text { Direct supervision }\end{array}$ \\
& $\begin{array}{l}\text { Training courses } \\
\text { Practice }\end{array}$ \\
\hline
\end{tabular}

GI, gastrointestinal; PM, postmortem.

tions, contraindications, and risks of the procedure. Practical skills, in addition to probe introduction, include positioning and manipulation of the probe to obtain all the required views and information and competence in interpreting the results of studies.

To date it has been fairly difficult to get much formal TOE training in the United Kingdom but this is increasingly available (table 5) as the number of trained operators increases, formal training courses emerge, and the literature (including text books, reviews, journals, and videos) on the subject expands. Use of all these resources will speed acquisition of skill.

\section{Safety, risks, and complications}

TOE is safe when it is performed carefully by trained staff. Nonetheless, complications can and do occur (table 6) and it is important not only to know what they may be but also to know how to avoid them.

In a recent large multicentre study of experience in 15 European centres with 10419 TOE examinations insertion of the probe was unsuccessful in $1.9 \%$ and the procedure was interrupted despite successful insertion in $0.88 \%$ (usually because the patient could not tolerate the probe or because cardiopulmonary or bleeding complications developed). ${ }^{2}$ There was one death (from gastrointestinal bleeding) (mortality $0.0098 \%$ ). In addition, a diabetic woman has died of cardiorespiratory arrest 5-10 minutes after the procedure. ${ }^{3}$ As might be expected, mortality and morbidity are slightly higher than for routine diagnostic endoscopy of the upper gastrointestinal tract $(0 \cdot 004 \%$ and $0 \cdot 13 \%$ respectively ${ }^{4}$ ).

In addition to mechanical injury TOE can cause pressure damage at the site of contact or thermal injury by local heat generation.

\section{Table 6 Complications of TOE}

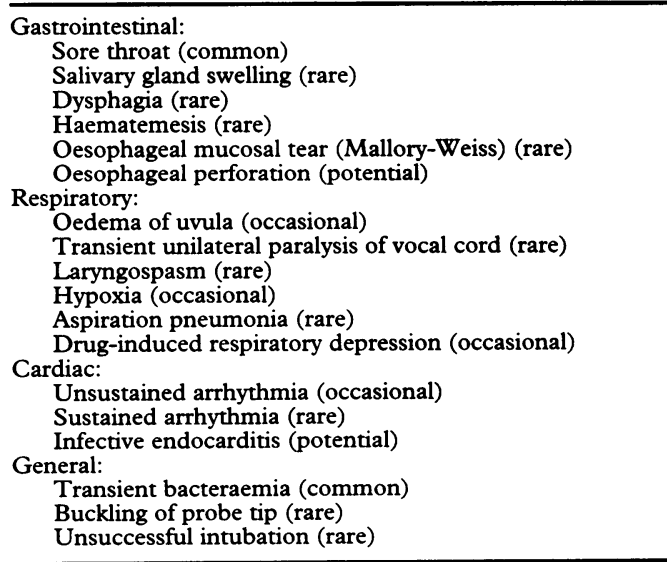

However, in practice these complications are rare. Table 6 lists most known complications; some are worth mentioning here. The most feared (but as yet unreported) complication of TOE is perforation of the oesophagus at its junction with the hypopharynx or at its lower end and particularly where there is an oesophageal tumour. When there is a history of dysphagia TOE should not be performed until the patient has been investigated fully. Insertion of the probe should never be forced and should always be synchronised with swallowing.

Bacteraemia occurs after $7-16 \%$ of TOE intubations ${ }^{56}$ and $4-13 \%$ of diagnostic endoscopy examinations of the gastrointestinal tract. ${ }^{7}$ The organisms involved are the predictable ones-Staphylococcus aureus, Staphylococcus epidermidis, and alpha haemolytic streptococci. However, there has been only one report of a case of streptococcal endocarditis temporally related to $\mathrm{TOE}^{8}$ and none causally or directly linked to TOE. Therefore the routine use of antimicrobial prophylaxis before TOE is not advised currently even in high risk patients.

Significant sustained arrhythmias are rare and transient; and minor ones occur in less than $1 \%$ of cases. Changes in heart rate and blood pressure are generally small and well tolerated. There is little evidence of myocardial ischaemia during or after the procedure.

\section{Conclusion}

TOE is a relatively non-invasive investigation that can produce high quality images of great diagnostic value. It is valuable in a wide range of indications and is an appropriate service in most medical institutions including DGHs, where it will improve the diagnosis of conditions such as aortic dissection that are difficult to diagnose by other means and aid decision making regarding the need for specialist referral to a cardiac centre. The successful development of such a service requires consideration of several factors including equipment needs, location, staffing, training, policies and procedures, and education of patients. With appropriate planning and attention to detail a laboratory can be set up and a high quality programme developed surprisingly quickly and efficiently.

1 Pearlman AS, Gardin JM, Martin RP, Parisi AF, Popp RI Quinones MA, Stevenson JG, Schiller NB, Seward JB, Stewart WJ. Guidelines for physician training in transoesophageal echocardiography: Recommendations of the oesophageal echocardiography: Recommendations of the American Society of Echocardiography Committee for Physician Training in Echoc

2 Daniel WG, Erbel R, Casper W, et al. Safety of transaniel WG, Erbel R, Casper W, et al. Safety of transoesophageal echocardiography. A multicentre sur.

3 Ansari A. Transesophageal two dimensional echocardiogra phy: Current perspectives. Prog Cardiovasc Dis 1993; phy: Curre

4 Silvis SE, Nebel O, Rogers G, et al. Endoscopic complications. Results of the 1974 American Society for Gastrointestinal endoscopy survey. $¥ A M A$ 1976;235: 928-30.

5 Gorge G, Erbel R, Heinrichs KJ, et al. Positive blood cultures during transoesophageal echocardiography. $A m$ f Cardiol 1990;65:1404-5.

6 Dennig K, Sedlmayr V, Selling B, et al. Bacteraemia with transoesophageal echocardiography. Circulation 1989; 80(suppl 2):473.

7 Botoman VA, Surawicz CM. Bacteraemia with gastrointestinal endoscopic procedure. Gastrointest Endosc 1986 32:342-6.

8 Foster E, Kusumoto FM, Sobols M, et al. Streptococca endocarditis temporally related to transesophagea echocardiography. F Am Soc Echocardiogr 1990;3:424-7. 